\title{
Assessment of a GNSS/INS/Wi-Fi \\ Tight-Integration Method Using Support Vector Machine and Extended Kalman Filter
}

\author{
Marco Mendonça and Marcelo C. Santos
}

\begin{abstract}
Wi-Fi derived positions have been used in the past few years as a complementary source of positioning information for GNSS and Inertial Systems (INS). Ubiquitous positioning that transitions from indoors to outdoors and vice-versa is currently a hot topic of research. In this context, this study aims to analyze the potential of directional antennas sequentially tracking Wi-Fi signals on the 11 channels around the $2.4 \mathrm{GHz}$ frequency in order to serve as an integrated signal for GNSS and INS positioning. Considering, as an example, a single point positioning (SPP) strategy coupled with an INS, the use of directional antennas can be beneficial in order to provide absolute directions of travel by the means of a Support Vector Machine (SVM) lane matching. In order to test the given hypothesis, real-world experiments were performed in areas with and without obstruction in an urban environment. Using a post-processed, smoothed in both forward and backward modes, and finally edited postprocessed kinematic (RTK) solution as a reference, the solution integrating SPP GNSS, INS and Wi-Fi was assessed in terms of accuracy. Preliminary results show that such a combination of the directional antennas along with GNSS and INS and their respective SVM and EKF filters, can provide sub-meter accuracy at all times without the need of precise orbits or differential corrections, increasing solution availability, reliability and accuracy on a scalable and cost-effective way.
\end{abstract}

\section{Keywords}

GNSS $\cdot$ INS $\cdot$ Sensor integration $\cdot$ SVM $\cdot$ Vehicle navigation $\cdot$ Wi-Fi

\section{Introduction}

Sensor integration techniques are a contemporary topic of research, since mobile platforms can now achieve the computational power required for such tasks. The use of Global Navigation Satellite Systems (GNSS) as a source of positioning, albeit widespread in applications, has limitations particularly noticeable in urban environments. The main damaging effects on GNSS positioning in urban environments are signal obstructions and reflections, causing problems

M. Mendonça $(\triangle) \cdot$ M. C. Santos

University of New Brunswick, Department of Geodesy and Geomatics

Engineering, Fredericton, NB, Canada

e-mail: marco.mendonca@unb.ca; msantos@unb.ca with both signal quality (usually yielding low signal-tonoise ratios), and low number of visible satellites. Several studies have been performed in order to quantify, analyse, and overcome such limitations using different techniques, such as solutions using new GNSS signals (Hsu et al. 2015), novel mathematical models to constrain the accumulating INS errors (Grejner-Brzezinska et al. 2001), sensor integration techniques, and signal-of-opportunity concepts (Groves 2011). With the growing demand for accurate and reliable urban positioning fueled by the advent and popularization of autonomous vehicles, improvements in this area are not only of academic value, but also of immediate practical applications. In this context, the cost and processing power requirements of the solutions are of paramount importance. Accurate and ubiquitous positioning equipment, such as 
GNSS/INS integrated NovAtel SPAN ${ }^{\circledR}$, may have comparable costs to a semi-autonomous vehicle, such as the Tesla Model 3, therefore, not being capable of reaching massmarket applications.

With the aforementioned situation in mind, and considering the challenges of positioning in urban areas, the integration of cost-effective sensors and improvements in mathematical models are viable and tested alternatives to overcome such challenges (Grejner-Brzezinska et al. 2001; Groves 2008). In this study, a combination of Wi-Fi signals recorded by directional antennas, an INS and a GNSS (GPS + GLONASS) receiver is studied in order to asses how information from directional Wi-Fi antennas can be integrated in a GNSS/INS solution and what is the benefit of it. The remainder of this paper is divided among the following sections: a brief review of traditional GNSS/INS integration techniques, an overview of Wi-Fi positioning techniques, the development of an integration technique between the three systems, experiment design, and, finally, results and conclusions.

\section{GNSS/INS Integration Techniques}

Amongst several possible ING/GNSS integration techniques (Groves 2008), in this paper, the loosely-coupled integration is explored and used as basis for the integration with Wi-Fi derived information. Figure 1 shows an overview of how the loosely-coupled integration is performed. Block 1 represents a traditional GNSS positioning filter. This filter can output positions, velocity and timing (PVT) from any positioning method, such as single point positioning (SPP), precise point positioning (PPP) or real-time kinematic (RTK). Block 2



Fig. 1 Overview of a loosely-coupled integration scheme represents an INS mechanization method, where positions are integrated to a current epoch using acceleration and angular velocity measurements. Without a long-term source of stability and constraint and due to the integrative nature of the INS mechanization procedure, errors rapidly accumulate, making the propagated coordinate unusable due to a high and exponentially growing bias. Referring again to Fig. 1, a closed-loop solution is estimated within Block 2. Finally, on Fig. 1, Block 3 represents the integration procedure of GNSS-derived coordinates and INS-derived displacements. A Kalman filter with the following state vector was then implemented on Block 3:

$$
x_{t}=\left[r_{3}, v_{3}, \Psi_{3}, b_{a}, b_{g}\right] \text {, }
$$

where the following vectors are represented: $r_{3}$ for position, $v_{3}$ for velocity, $\Psi$ for vehicle attitude, and $b_{a}$ and $b_{g}$ for accelerometer and gyroscope biases, respectively. The measurement vector $z_{t}$ is given as:

$$
\begin{array}{r}
z_{t}^{L C}=\left[\Delta r_{x}, \Delta r_{y}, \Delta r_{z},\right. \\
\left.\Delta v_{x}, \Delta v_{y}, \Delta v_{z}\right]
\end{array}
$$

where $\Delta r$ is the difference between the estimated coordinates from GNSS and the propagated coordinates from INS at the same epoch on each axis, and the equivalent for velocity in $\Delta v$.

\section{Outdoor Wi-Fi Positioning}

In general lines, an outdoor Wi-Fi positioning system is comprised of two phases: training and positioning. Figure 2 shows a Wi-Fi positioning system as developed by Lu et al. (2010). In this system, which served as a basis in this study, a survey of a predefined route is performed. In this route, coordinates of the reference points (RP) are estimated and the local Wi-Fi networks (a mixture of public and private access points) received signal strength (RSS) are scanned and stored. The selection of the reference points is based on the latency of the Wi-Fi scanning. In this study, on average, one point was scanned at every $1.2 \mathrm{~s}$ in multiple passes over the same lane. With the data acquired during the training phase, a one-class support vector machine (SVM) unsupervised classifier is applied to assess if the clusters of RSS measurements represent properly the different lanelets. Once this capability is identified, a standard SVM classifier is then trained in order to optimally identify sections with similar RSS measurements. Finally, each one of those sections is linked to a lanelet (Bender et al. 2014), by an optimal non-linear SVM classifier (Steinwart and Christmann 2008). The result of this system is a trained model that is able 




Fig. 2 Overview of a Wi-Fi/GNSS integration method (Lu et al. 2010)

to identify, given a set of measurements from the Wi-Fi antennas $z_{t}=\left[M A C_{1}, R S S_{1} ; \ldots M A C_{n}, R S S_{n}\right]$, to which lanelet it most likely belongs. Since the number of RSS observations varies from area to area, only the 20 strongest measurements are used as an input for the SVM classifier. In case less observations are present, the dataset is padded with zeros, up to a lower limit of five observations. These values were empirically determined during the one-class SVM clustering. Figure 3 shows a section of the surveyed area. In this example, each numbered lanelet has three attributes: azimuth, maximum posted speed and average GNSS Geometry Dilution of Precision (GDOP), roughly representing how well the GNSS solution is expected to be in this region. Each lanelet has the empirical limit of two blocks of length, or if one of the three mentioned properties is significantly different at a certain point forward. It is important to notice that in this system, the absolute location derived from the Wi-Fi RSS measurements is not important, as long as the correct lanelet is identified and its attributes are applied in the integration filter. This approach makes it possible to extract useful information from Wi-Fi signals that can be directly used in vehicle control systems.

\section{$4 \quad$ Integration Between GNSS, INS and Wi-Fi}

Given the rapid diverging characteristic of INS position propagation, in the absence of GNSS for a considerable period of time - situation common in urban areas - any

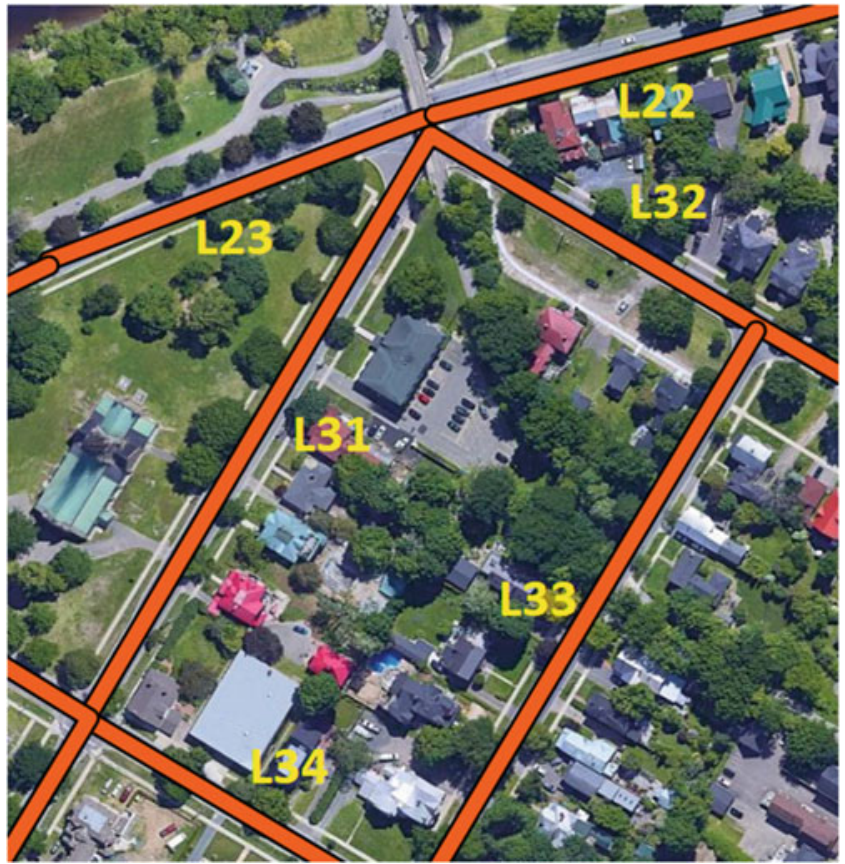

Fig. 3 Example of lanelets on a section of the surveyed area

system relying on this information may suffer from unreliable position estimates. On an autonomous vehicle scenario, this situation may cause a disengagement of the auto-pilot or possibly accidents.

As an additional information, a radio map (equivalent to the database in Fig. 2) was built based on the work by $\mathrm{Lu}$ 




Fig. 4 Overview of the directional antenna Wi-Fi data collection system

et al. (2010) with an added layer of post-processing and editing for improved accuracy. This database is derived from the schematic on Fig. 4. Once the SVM model estimates a probable match for the measurements $z_{t}$, information is then extracted from that region's lanelet and integrated into the GNSS/INS loosely-coupled filter during the application of the non-holonomic constraint (NHC) (Groves 2008). The $\mathrm{NHC}$ is applied after the main integration filter and is responsible for constraining the vehicle movement on forwards. The cross-track and up velocities are constrained as zero, and any movement in those directions is then treated as a measurement residual during the filtering. The NHC is applied by the means of a Kalman filter with the measurement vector as:

$$
z_{t}^{N H C}=\left[v_{c} v_{u p}\right],
$$

where $v_{c}$ and $v_{u p}$ are the cross-track and up velocities, respectively, estimated during the loosely-coupled integration, and partnered with the following design matrix:

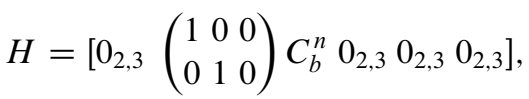

where $C_{b}^{n}$ is the rotation matrix between the body-frame (same reference as $z_{t}$ ) and the navigation (ENU) frame. It is possible to see on Eq. 5 that the body-to-navigation rotation matrix $C_{b}^{n}$ is a function of the travel direction angle yaw along with the vehicle's pitch and yaw. Retrieving the yaw angle from the Wi-Fi radio map, a vehicle motion constraint

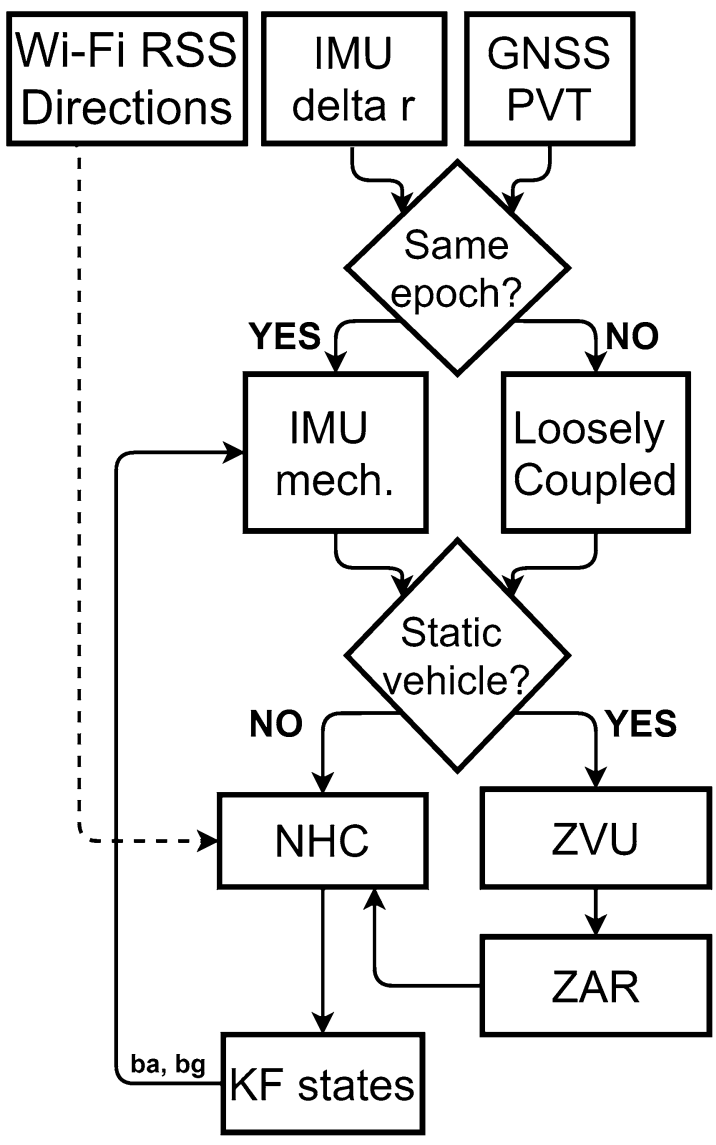

Fig. 5 Overview of the GNSS/INS/Wi-Fi integration algorithm

(Groves 2008) is then created and $C_{b}^{n}$ becomes (Rogers 2007):

$$
C_{b}^{n}=\left|\begin{array}{ccc}
c \psi c \theta & c \psi s \theta s \phi-s \psi c \phi & c \psi s \theta s \phi+s \psi c \phi \\
-s \psi c \theta & -s \psi s \theta s \phi-c \psi c \phi & c \psi s \phi-s \psi s \theta c \phi \\
s \theta & -c \theta s \phi & -c \theta c \psi
\end{array}\right|
$$

where $\phi, \theta$, and $\psi$ are the roll, pitch and yaw, or azimuth, angles respectively, and $c$ and $s$ are cosine and sine functions, also respectively.

By applying the roll and pitch estimates from the filter before the NHC and updating yaw, the system biases, along with all other estimates, are all updated with the external information. Figure 5 shows an overview of the integration methodology. The updated parameters after the NHC are then used as input for the INS mechanization, and updated again during the next INS/GNSS integration. Other techniques to integrate external yaw information to the filter have been explored in literature (Falco et al. 2013; 
Angrisano 2010), and will be investigated in future versions of this study.

\section{$5 \quad$ Experiment and Results}

In order to assess the use of the Wi-Fi derived information, an experiment was performed in Fredericton, NB, Canada. The experiment consisted of a 73-min drive in several areas with different satellite coverage, obstruction, and multipath characteristics. Figure 6 shows the equipment of top of the vehicle. Inside, right above the vehicle's rear axis, a KVH TG60000 tactical inertial system was deployed. Since the RSS measurements are observed by directional antennas and input in the SVM training phase, it is clear that antennas pointing in opposite directions will have different measurements for the same location. This represents an important assumption of this study, and more segmented information for the hyper-plane model to better cluster the input information.
For the SVM training algorithm, data from several previous surveys in the area were utilized. With a rate of $90 \%$ of training data for $10 \%$ of test, the accuracy of the classifier was $82 \%(\mathrm{~N}=7,422)$ on the correct lanelet. On a more detailed analysis of the misclassifications (about 130 points out of 7,422), it was found that they happened in situations where the vehicle was stopped at street crossings, and the classifier could not differentiate between the end of three or more lanelets. The misclassifications in this stance do not represent a risk for the system integrity, since sudden "jumps" from one lanelet to another can be easily ruled out by the INS measurements.

In this section, for brevity's sake, one particularly harsh section of the full experiment was selected to assess the method.

Figure 7 shows the analyzed route with the results from an edited post-processed kinematic (PPK) used as the reference. Finally, by forcing the integration with the proper yaw angle of the road, the solution is smoothed and kept in the driving lanes, as Fig. 10 shows. Figure 8 shows the results from a

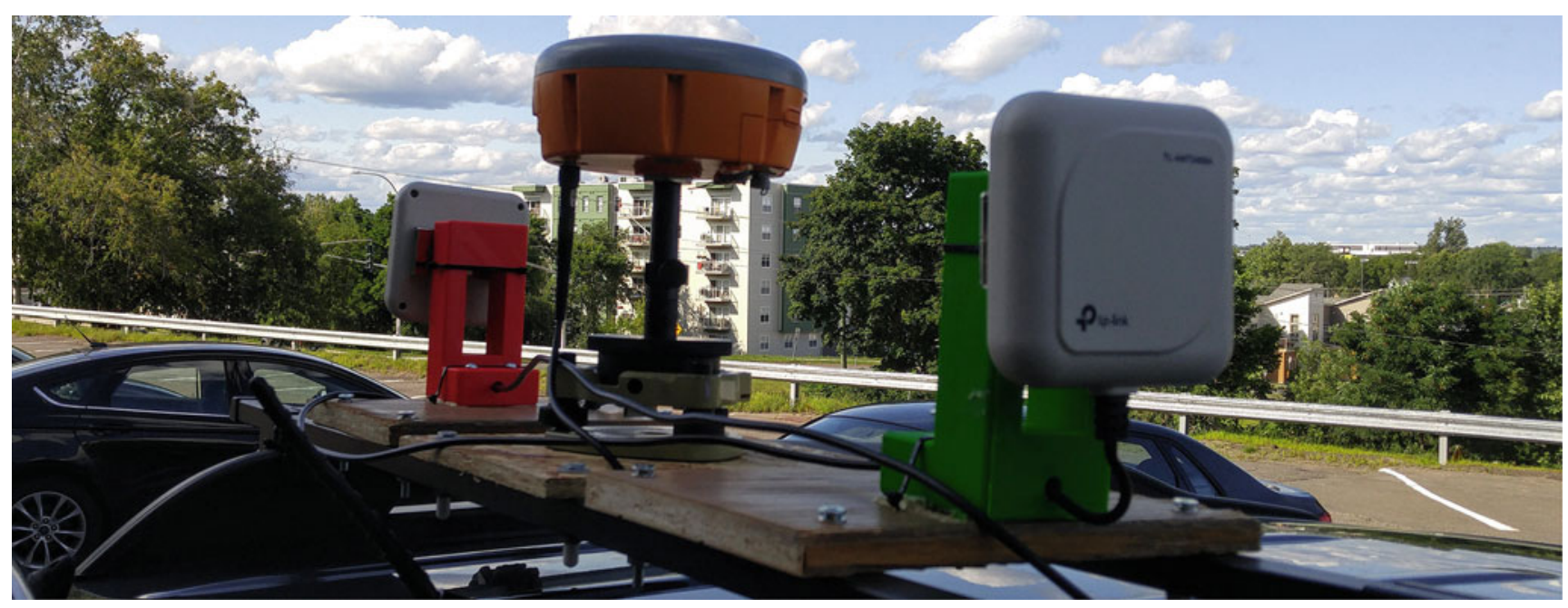

Fig. 6 Hardware of the initial test campaign. Septentrio APS3G multi-GNSS receiver on the center, and two TPLINK directional Wi-Fi (2.4 GHz band) antennas pointing sideways

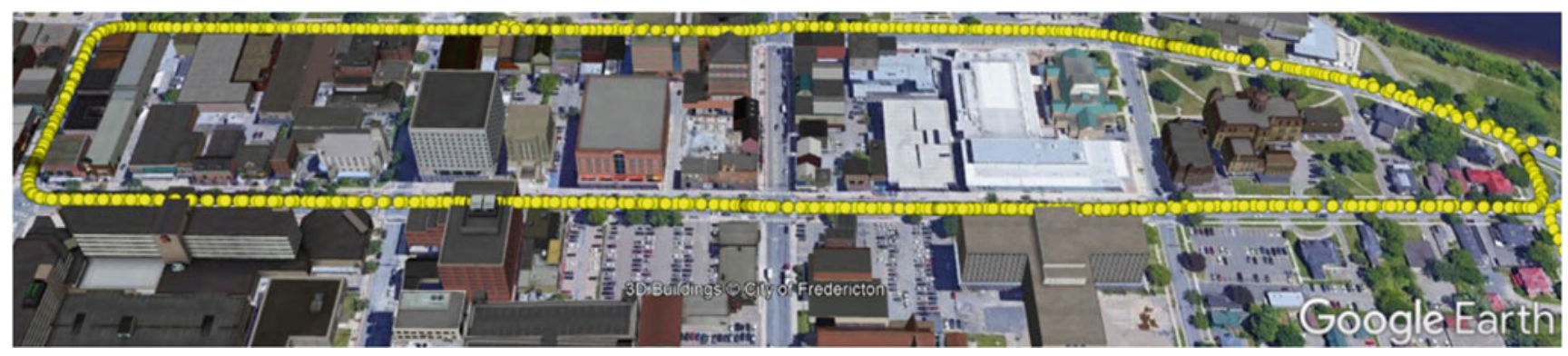

Fig. 7 Downtown Fredericton area with edited PPK reference shown in yellow 


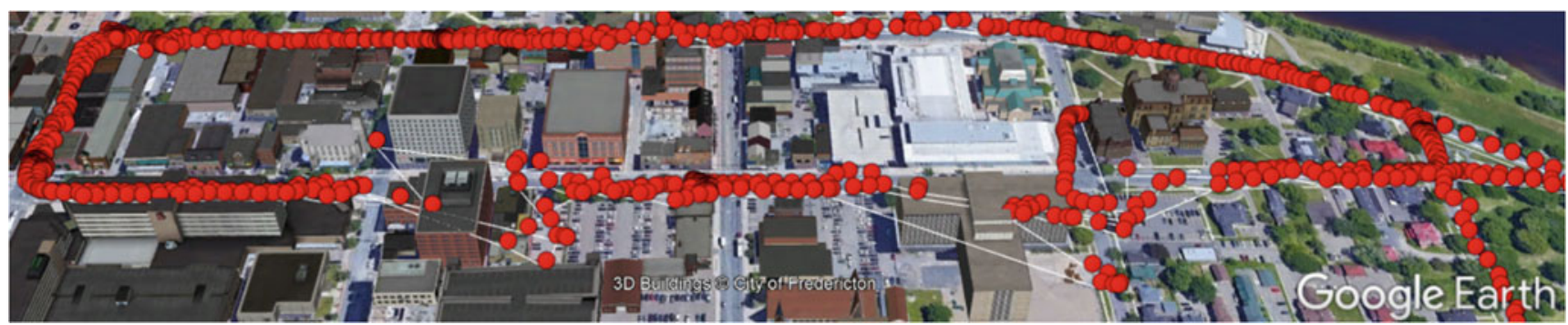

Fig. 8 SPP results in the test area

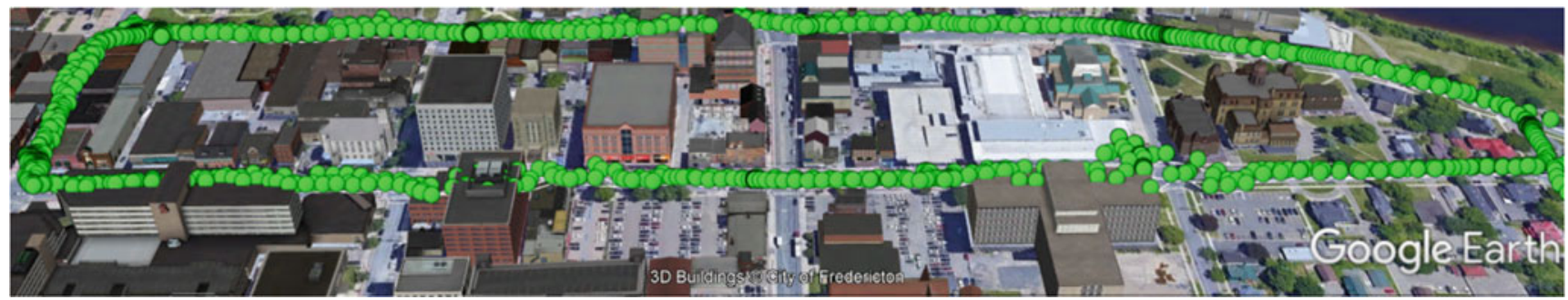

Fig. 9 SPP and INS loosely-coupled integration results

Table 1 Horizontal component RMS of the techniques evaluated versus post-processed RTK

\begin{tabular}{l|l}
\hline Technique & $R M S_{\text {HORZ }}$ \\
\hline SPP & $2.914 \mathrm{~m}$ \\
\hline SPP+INS & $1.219 \mathrm{~m}$ \\
\hline SPP+INS+Wi-Fi/SVM & $0.970 \mathrm{~m}$ \\
\hline
\end{tabular}

single-point positioning (SPP) in the same area as Fig. 7. The processing strategy utilized ion-free observables, a $15^{\circ}$ elevation mask, and Saastamoinen as tropospheric model. It is possible to see that in two particular areas on the bottom street, the number of satellites drops and the multipath effect is more pronounced, generating jumps in the trajectory. Figure 9 shows the results from a standard loosely-coupled integration in the same area. Even with criteria in place to avoid integration when the GNSS data is not reliable (number of satellites greater than 4 and reported horizontal standard deviation lower than $5 \mathrm{~m}$ ), in the areas where the absolute positions are not reliable, the integration itself returns values out of the driving lane, and out of the streets on some occasions.

Table 1 summarizes the RMS values using the PPK solution as reference. The RMS of the GNSS+INS+Wi-Fi/SVM integrated solution is $20 \%$ improved from the GNSS+INS only, and $66 \%$ better than the SPP solution only.

\section{Conclusions}

From Table 1 and Fig. 10, it is possible to see the already explored in literature effect of an external directional constraint on navigation. This paper explores the novel possibility of integrating an SVM classification on Wi-Fi RSS data to generate such directional constraints to be integrated in the filter. Future version of this study will explore other methods of integrating yaw measurements, and explore more fine system calibration techniques to improve the already promising results achieved. 


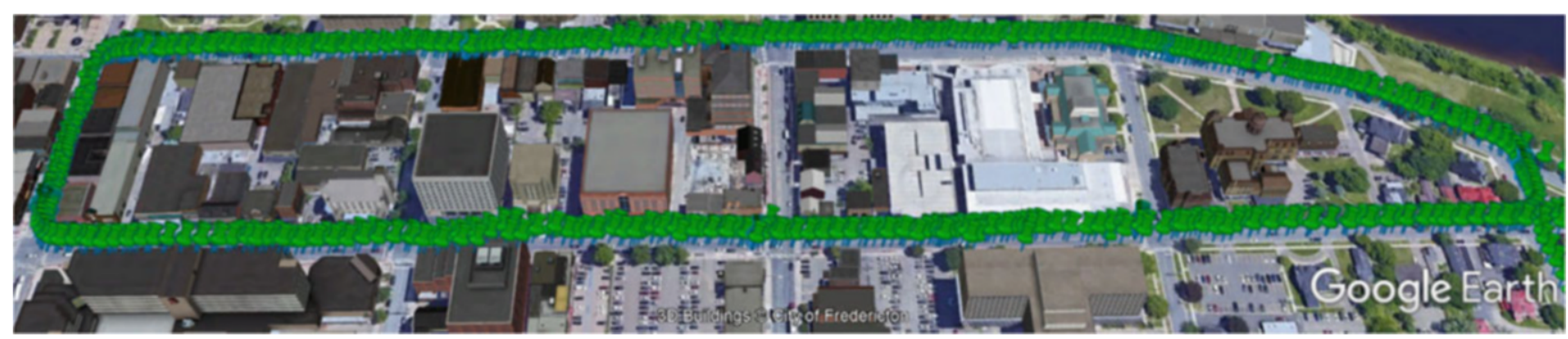

Fig. 10 SPP, INS and external yaw constraint on a loosely-coupled filter

\section{References}

Angrisano A (2010) GNSS/INS integration methods. Ph.D. thesis, Universita' Degli Studi Di Napoli

Bender P, Ziegler J, Stiller C (2010) Lanelets: efficient map representation for autonomous driving. In: IEEE intelligent vehicles symposium, proceedings (Iv), pp 420-425. https://doi.org/10.1109/ IVS.2014.6856487

Falco G, Campo-Cossío M, Puras A (2013) MULTI-GNSS receivers/IMU system aimed at the design of a heading-constrained tightly-coupled algorithm. In: 2013 International conference on localization and GNSS, ICL-GNSS 2013, June. https://doi.org/10. 1109/ICL-GNSS.2013.6577263

Grejner-Brzezinska DA, Yi Y, Toth CK (2001) Bridging GPS gaps in urban canyons: the benefits of ZUPTs. Navigation 48(4):216-226. https://doi.org/10.1002/j.2161-4296.2001.tb00246.x

Groves PD (2008) Principles of GNSS, inertial, and multisensor integrated navigation systems, vol. 2. Artech House, London
Groves, P.D.: Shadow matching: a new GNSS positioning technique for urban canyons. J Navig 64(3):417-430 (2011). https://doi.org/10. 1017/S0373463311000087

Hsu L-T, Gu Y, Chen F, Wada Y, Kamijo S (2015) Assessment of QZSS L1-SAIF for 3D map-based pedestrian positioning method in an urban environment. In: Proceedings of the 2015 international technical meeting of the Institute of Navigation, Dana Point, January 2015, pp 331-342

Lu H, Zhang S, Dong Y, Lin X (2010) A Wi-Fi/GPS integrated system for urban vehicle positioning. In: IEEE conference on intelligent transportation systems, proceedings, ITSC, pp 1663-1668. https:// doi.org/10.1109/ITSC.2010.5625268

Rogers RM (2007) Applied mathematics in integrated navigation systems, 3rd edn. https://doi.org/10.2514/4.861598

Steinwart, I., Christmann, A.: Support Vector Machines. Information Science and Statistics. Springer New York, New York, NY (2008). https://doi.org/10.1007/978-0-387-77242-4

Open Access This chapter is licensed under the terms of the Creative Commons Attribution 4.0 International License (http://creativecommons. org/licenses/by/4.0/), which permits use, sharing, adaptation, distribution and reproduction in any medium or format, as long as you give appropriate credit to the original author(s) and the source, provide a link to the Creative Commons licence and indicate if changes were made.

The images or other third party material in this chapter are included in the chapter's Creative Commons licence, unless indicated otherwise in a credit line to the material. If material is not included in the chapter's Creative Commons licence and your intended use is not permitted by statutory regulation or exceeds the permitted use, you will need to obtain permission directly from the copyright holder.

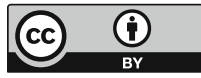

